\title{
Evaluation of Bovine Derived Xenograft Combined with Bioresorbable Collagen Membrane in Treatment of Intrabony Defects
}

\author{
Dr. Ranjita Shrestha Gorkhali, ${ }^{1}$ Dr. Shaili Pradhan, ${ }^{1}$ Dr. Rejina Shrestha, ${ }^{1}$ Dr. Shweta Agrawal, \\ Dr. Krishna Lamicchane, ${ }^{1}$ Dr. Pramod Kumar Koirala, ${ }^{1}$ Dr. Benju Shrestha ${ }^{1}$ \\ ${ }^{1}$ Periodontology and Oral Implantology Unit, Department of Dental Surgery, \\ Bir Hospital, National Academy of Medical Sciences, Kathmandu, Nepal.
}

\begin{abstract}
Introduction: Treatment of periodontal diseases done by surgical therapy depends upon extent and severity of disease. The ultimate goal of periodontal reconstructive surgery is to regenerate tissues destroyed during periodontal disease.

Objective: To evaluate the effectiveness of bovine-derived xenograft with collagen membrane in treatment of intrabony defects by comparing it with open flap debridement alone.

Methods: This non-randomised controlled trial was conducted after ethical clearance, at Bir hospital from 2018 March to 2019 April. The study recruited 38 patients by convenience sampling, age from 25-44 years, with chronic periodontitis, and willing to sign informed consent. Intrabony defects were treated by open flap debridement with bovine-derived xenograft and bioresorbable collagen membrane (Test group) and open flap debridement alone (Control group). Probing pocket depth, clinical attachment level, gingival recession, oral hygiene status, and gingival status were assessed at baseline and six months.

Results: Six months after therapy, in Test group probing pocket depth reduction was $5.2 \mathrm{~mm}$ and gain in mean clinical attachment level was $4.3 \mathrm{~mm}$. In Control group, mean probing pocket depth reduction was $3.8 \mathrm{~mm}$ and mean gain in clinical attachment level was $2.7 \mathrm{~mm}$. The test treatment resulted in statistically higher probing pocket depth reduction and clinical attachment level gain than Control group.

Conclusion: Both therapies resulted in significant probing pocket depth reductions and clinical attachment gains, and treatment with open flap debridement with bovine-derived xenografts and collagen membrane resulted in significantly higher probing pocket depth reduction and clinical attachment gain than treatment with open flap debridement alone.

Keywords: Bioabsorbable collagen membrane; bovine-derived xenograft; intrabony defects; open flap debridement; regenerative periodontal therapy.
\end{abstract}

\section{INTRODUCTION}

Periodontal disease is a chronic infectious disorder caused by bacterial plaque characterised by destruction of toothsupporting tissue. ${ }^{1}$ Periodontal diseases can be treated by non-surgical periodontal therapy and surgical therapy depending upon extent and severity of disease. Surgical periodontal therapy with use of bone grafts along with

\footnotetext{
Correspondence:

Dr. Ranjita Shrestha Gorkhali

Periodontology and Oral Implantology Unit, Department of Dental Surgery, Bir Hospital, National Academy of Medical Sciences, Kathmandu, Nepal.

email: ranjish2@yahoo.com

Citation

Gorkhali RS, Pradhan S, Shrestha R, Agrawal S, Lamicchane K, Koirala PK, et al. Evaluation of Bovine-derived Xenograft Combined with Bioresorbable Collagen Membrane in Treatment of Intrabony Defects. J Nepal Soc Perio Oral Implantol. 2020 Jul-Dec;4(8):61-7.
}

or without collagen membranes are effective treatment modalities.

Guided tissue regeneration (GTR) is more effective than an open flap debridement (OFD) alone. ${ }^{2}$ Membrane placement is based on biological principles of GTR which prevents apical migration of gingival epithelial cells during initial stages of healing allowing periodontal ligament cells to repopulate root surface. ${ }^{3}$ GTR is combined with bone grafts to support barrier material and to prevent it from collapsing into defect or onto root. Deproteinised bovine bone mineral (DBBM) has been widely used with adjunct to GTR. Animal studies ${ }^{4}$ and clinical cases ${ }^{5}$ suggest that healing following GTR with DBBM is partly characterised by periodontal regeneration. Bovine-derived xenograft (BDX) which possesses good osteoconductive properties has been introduced as a grafting material for regenerative periodontal surgery. ${ }^{5-6}$

The aim of this study was to evaluate the effectiveness of BDX and collagen membrane (CM) in the treatment of intrabony defects by comparing it with open flap debridement alone. 


\section{METHODS}

This non-randomised controlled trial was conducted at Periodontology and Oral Implantology Unit, Department of Dental Surgery, Bir hospital, National Academy of Medical Sciences (NAMS), Kathmandu, Nepal from 2018 March to 2019 April. Ethical clearance was obtained from institutional review board, NAMS (Reg. no. 1145). The patients visiting the dental outpatient department (OPD) were included.

The sample size was calculated by using using the formula

$\mathrm{n}=\frac{2\left(\mathrm{Z}_{\alpha}+\mathrm{Z}_{\beta}\right)^{2} \mathrm{~S}^{2}}{\mathrm{~d}^{2}}=14.49$

$\mathrm{n}=$ sample size in each group

$\mathrm{z}_{\alpha}=1.96$ at $95 \%$ confidence level

$\mathrm{z}_{\beta}=0.84$ at $80 \%$ power

$\mathrm{S}=(\mathrm{S} 1+\mathrm{S} 2) / 2=(0.7+1.8) / 2=1.25$

$\mathrm{d}=(\mathrm{m} 1-\mathrm{m} 2)=(3.9-5.2)=-1.3$

using the mean and standard deviation values of probing depth at one year among the test and control groups were taken from Sculean et al. ${ }^{7}$ To adjust for dropout a minimum of 19 subjects in each group (a total of 38 individuals) were enrolled in the study. The 19 participants in Test group of Intrabony Defect (IBD) were treated with OFD+BDX+CM and 19 participants in Control group in IBD were treated with OFD alone.

Nonprobability (convenience) sampling was done which means those who meet the inclusion criteria were included. The inclusion criteria were patients of 25 years to 44 years undergoing periodontal therapy, presence of interproximal IBD more than or equal to $3 \mathrm{~mm}$ deep (distance between alveolar crest and base of the defect on intraoral periapical radiograph, IOPAR), interproximal probing pocket depth (PPD) more than or equal to $5 \mathrm{~mm}$ following phase I therapy (scaling and root planing, SRP), two and three walled bony defects.

The exclusion criteria were patients with present or past systemic illness that are known to affect the outcomes of periodontal therapy, immune compromised patients, pregnant and lactating females, smokers/those using any

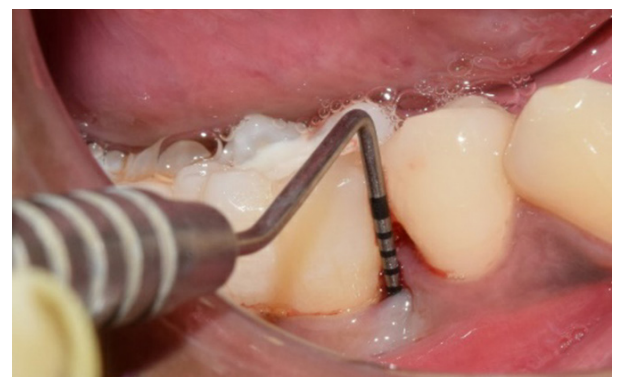

Figure 1: Baseline probing pocket depth buccally=10 $\mathrm{mm}$. other tobacco products, patients taking medications that may interfere with wound healing, those allergic to other medications, those having unacceptable oral hygiene, if plaque index (PI) is more than or equal to three after the re-evaluation of phase I therapy, and uncooperative patient.

Ethical clearance was obtained from the Institutional Review Board, NAMS, Bir Hospital. Written informed consent was obtained from each participant of the study and information were recorded by means of a proforma prepared for the study. The clinical examination of the patient was carried out with mouth mirror and University of North Carolina-15 (UNC - 15) periodontal probe.

All clinical examinations and non-surgical treatment were performed by one examiner and verified the eligibility of all subjects and ensured that all necessary pretreatment preparations had been carried out. Emergency treatment such as extraction, caries stabilisation, and initial endodontic therapy, if necessary, were completed before the non-surgical periodontal treatment. Before surgery, each patient was given careful instructions on proper oral hygiene measures. Following four weeks of phase I therapy, periodontal evaluation was performed to confirm the suitability of the sites for this study. The selected sites were divided into Test Group (OFD+BDX+CM) and Control Group (OFD alone) as per convenience.

Following administration of local anaesthesia, buccal and lingual sulcular incisions were made and mucoperiosteal flaps were elevated. Care was taken to preserve as much interproximal soft tissue as possible. Meticulous defect debridement and root planing were carried out with the use of Gracey curettes. A Case of intrabony defect treated with open flap debridement along with bovine derived xenograft and collagen membrane is shown in figures from Figure 1-10. Whereas a case of open flap debridement alone with customised stent has been depicted in Figure 11 and Figure 12.

No osseous recontouring were performed. The defects were filled with bovine porous bone mineral granules of particle size 0.25-1.0 mm (BDX, BioOss, Geistlich, Wolhusen, Switzerland) followed by placement of a bioresorbable collagen membrane of porcine origin (BioGide Perio, Geistlich, Wolhusen, Switzerland) as shown in Figure 5 and 6.

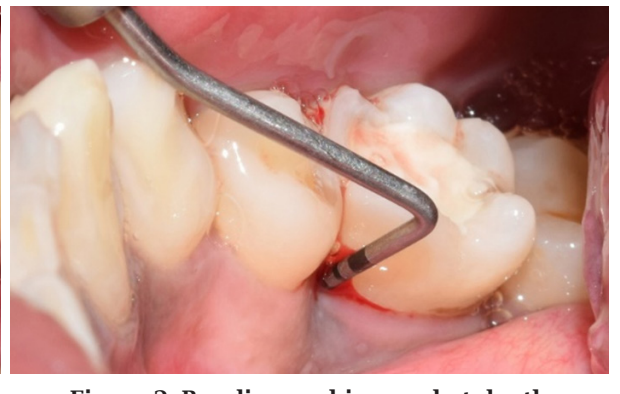

Figure 2: Baseline probing pocket depth lingually=12 $\mathrm{mm}$. 


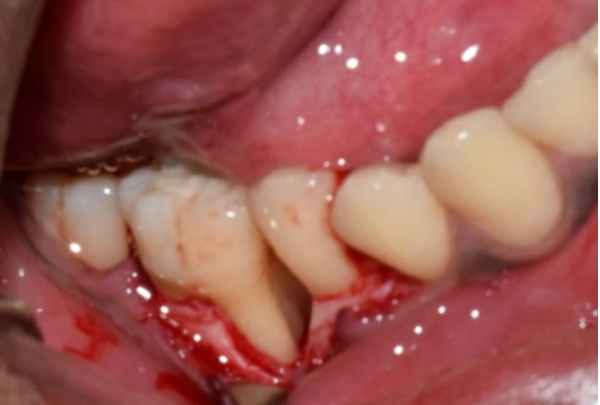

Figure 3: Intrabony defect.

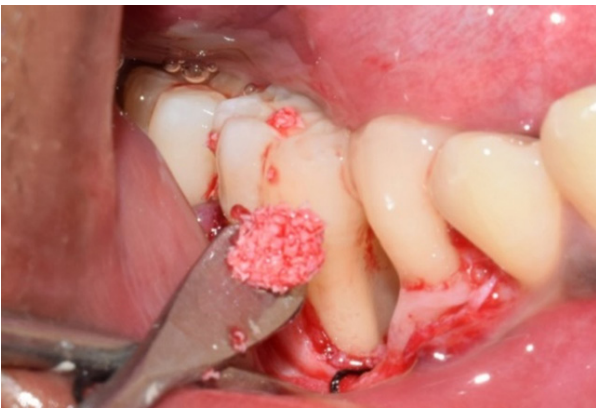

Figure 5: Bovine-derived xenograft placement.

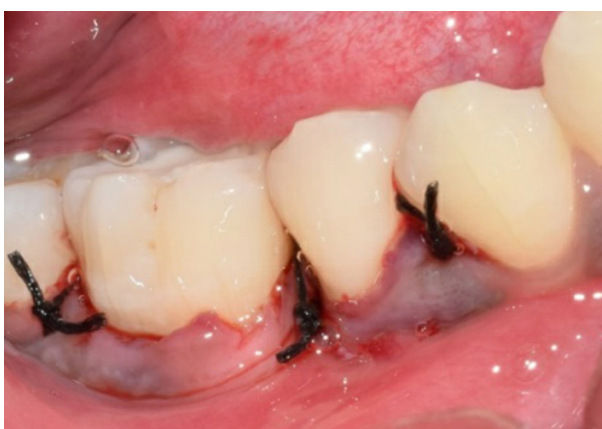

Figure 7: Suture placement.

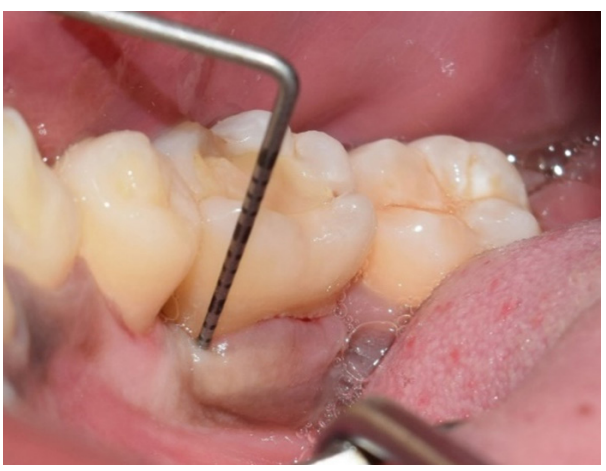

Figure 9: Probing pocket depth reduced to $2 \mathrm{~mm}$ at six months follow-up.

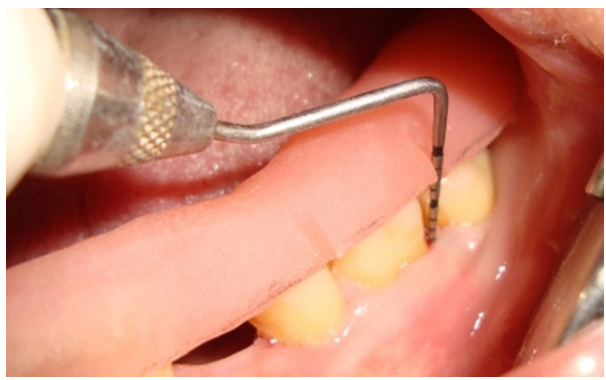

Figure 11: Probing pocket depth at baseline.

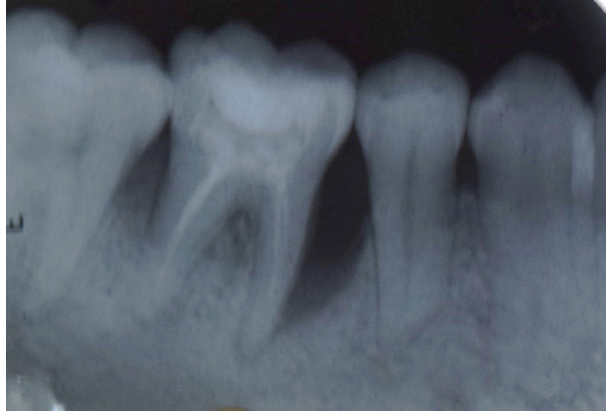

Figure 4: IOPAR Preoperative.

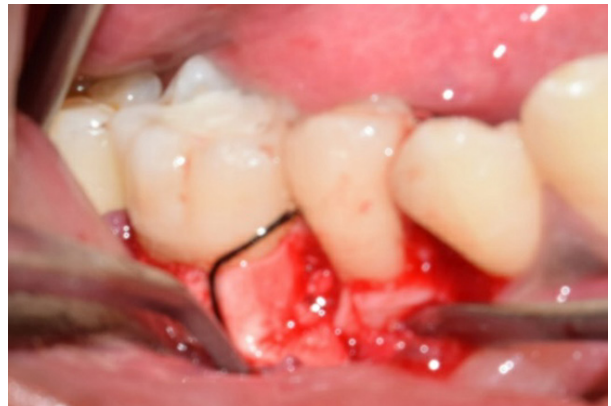

Figure 6: Adaptation of resorbable collagen membrane.

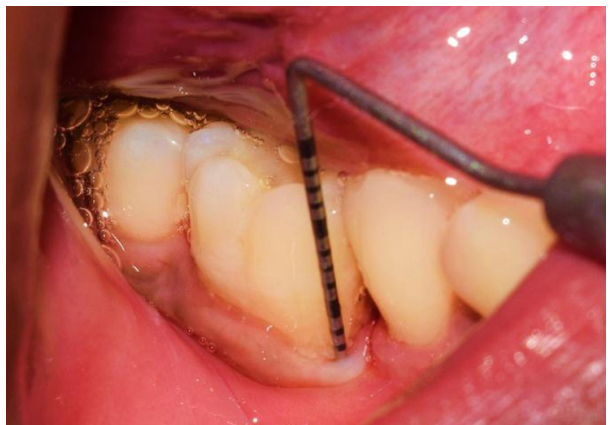

Figure 8: Probing pocket depth reduced to $3 \mathrm{~mm}$ at six months follow-up.

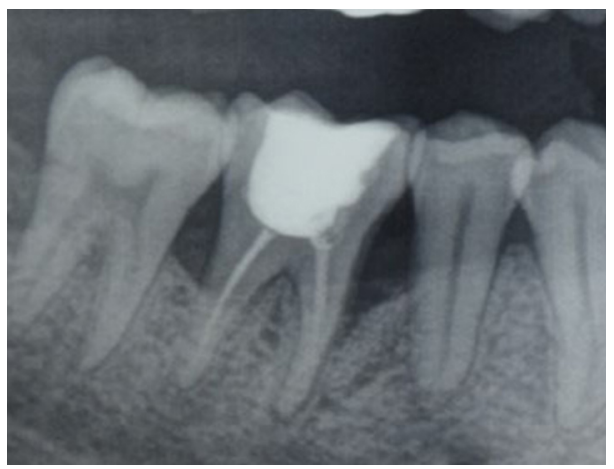

Figure 10: Bone fill can be observed in postoperative IOPAR.

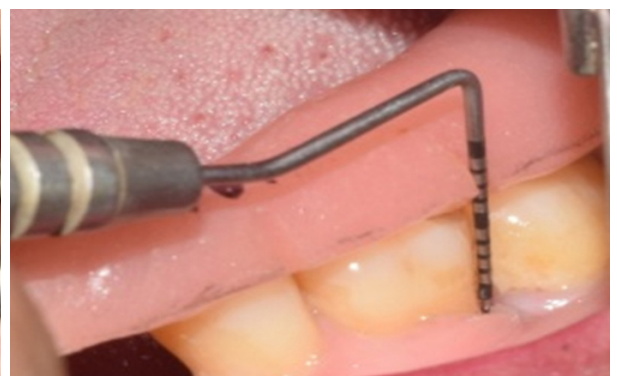

Figure 12: Probing pocket depth at follow-up at six months. 
To avoid the displacement, a silk suture was passed through the buccal and lingual flap before the placement. The mucoperiosteal flaps were repositioned and secured in place using 3-0/4-0 non-absorbable black silk surgical suture. Interrupted sutures were placed.

Antibiotics were prescribed after surgery to all patients. Capsule Amoxicillin (500 mg) was given three times daily for five days. The postoperative care consisted of $10 \mathrm{ml}$ of $0.2 \%$ chlorhexidine rinses twice a day for two weeks. The sutures were removed 7-14 days after the surgery depending upon the case. Recall appointments were scheduled every second week during the first two months after surgery and monthly following the rest of the observation period. Probing or subgingival instrumentation were not performed during the first year after surgery.

The clinical parameters measured were: PPD - measured from the gingival margin to the base of the pocket and clinical attachment level (CAL) - measured from the cementoenamel junction (CEJ) to the base of the pocket. Other parameters assessed were Gingival Recession (GR), Plaque Index and Gingival Index (GI). Gingival Recession was measured from CEJ to gingival margin. Plaque Index was assessed according to Silness and Loe (1964) criteria, Gingival Index on the basis of Loe and Silness (1967) criteria. Clinical parameters were assessed at baseline before surgical therapy and six months after surgical therapy.

Patients were called for regular follow-up and motivated for maintaining a proper oral hygiene. Customised acrylic stents with grooves were used to ensure a reproducible placement of periodontal probe.
The data analysis was done with IBM SPSS Statistics for Windows, version 20 (IBM Corp., Armonk, N.Y., USA). $\mathrm{P}$ value was calculated under the predetermined level of significance (0.05) and confidence interval of 95\% was constructed. Chi-square test (continuity correction, exact test) was performed for qualitative or categorical variables. Normality of variables was checked with K-S test. Independent t-test was used to compare mean value across groups and mean value of difference between parameters at baseline and six months.

\section{RESULTS}

There were total 38 patients among which 19 were allocated for OFD with $\mathrm{BDX}+\mathrm{CM}$ and 19 for OFD alone (Table 1). According to WHO criteria, age group from 25 years (yrs) to 44 years were included in the study. Maximum number of patients were in 35-44 yrs age group. The mean age was 38.9 in OFD (Control group ) and 36.6 in OFD with BDX+CM (Test group).

Mean PPD reduction in Test Group was from $7.9 \mathrm{~mm}$ to 2.7 $\mathrm{mm}$, difference was $5.2 \mathrm{~mm}$ from baseline to six months whereas in Control Group mean PPD reduction was from 7.0 $\mathrm{mm}$ to $3.2 \mathrm{~mm}$, difference was $3.8 \mathrm{~mm}$ (Figure 13).

Mean gain in CAL in Test group was from $7.6 \mathrm{~mm}$ to 3.3 $\mathrm{mm}$, difference was $4.3 \mathrm{~mm}$ and in Control group it was from $7.3 \mathrm{~mm}$ to $4.6 \mathrm{~mm}$, difference was $2.7 \mathrm{~mm}$ which was almost twice more than the gain in Control group (Figure 14). Mean value of CAL at six months was statistically significant (Table 2). Mean value of difference in PPD and CAL at baseline and six months in Control and Test Group were statistically significant (Table 3).

Table 1: Demographic distribution among control group and test group, $\mathbf{n}(\%)$.

\begin{tabular}{|l|c|c|c|}
\hline \multicolumn{1}{|c|}{ Sex } & Control (n=19) & Test Group (n=19) & P value \\
\hline Female & $11(57.9)$ & $9(47.4)$ & 0.516 \\
\hline Male & $8(42.1)$ & $10(52.6)$ & \\
\hline
\end{tabular}

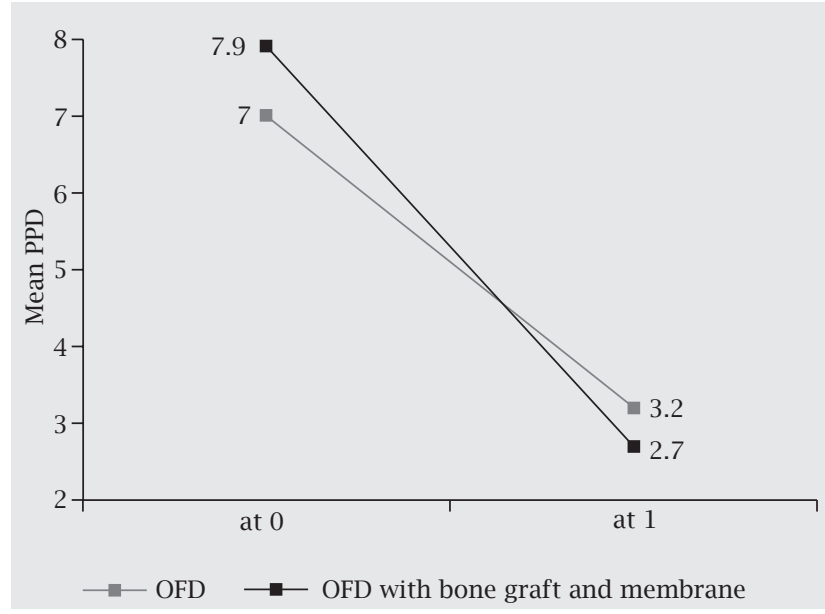

Figure 13: Comparison of mean probing pocket depth reduction in control group and test group at baseline and at six months.

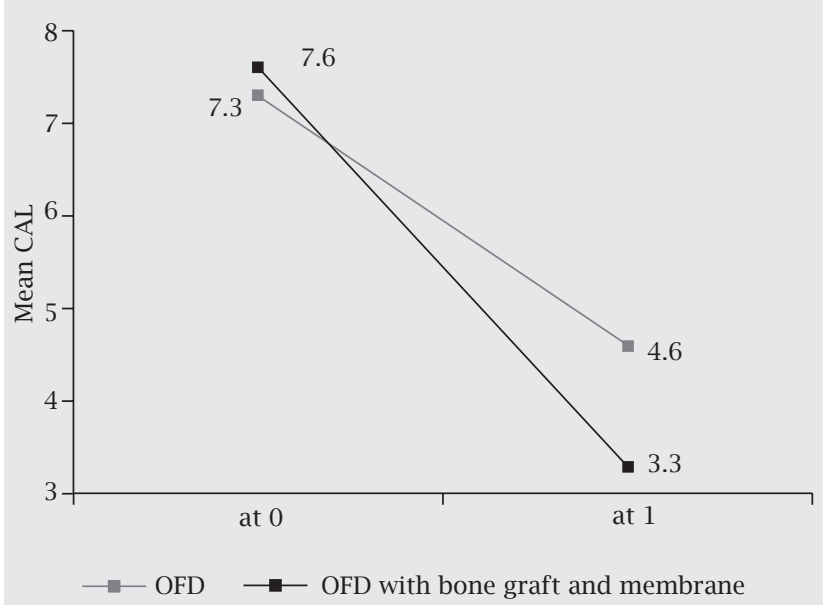

Figure 14: Comparison of mean clinical attachment level gain in control group and test group at baseline and at six months. 
Table 2: Comparison of age and periodontal parameters in control group and test group.

\begin{tabular}{|l|c|c|c|}
\hline & $\begin{array}{c}\text { Control Group (n=19) } \\
\text { Mean } \pm \text { SD }\end{array}$ & $\begin{array}{c}\text { Test Group (n=19) } \\
\text { Mean } \pm \text { SD }\end{array}$ & P value \\
\hline Age & $38.9 \pm 6.6$ & $36.6 \pm 5.2$ & 0.236 \\
\hline PPD-0 & $7 \pm 1.2$ & $7.9 \pm 1.5$ & 0.052 \\
\hline PPD-1 & $3.2 \pm 1.0$ & $2.7 \pm 0.9$ & 0.135 \\
\hline CAL-0 & $7.3 \pm 2.8$ & $7.6 \pm 1.7$ & 0.627 \\
\hline CAL-1 & $4.6 \pm 1.8$ & $3.3 \pm 1.6$ & 0.024 \\
\hline GR-0 & $1.2 \pm 1.2$ & $0.5 \pm 0.9$ & 0.053 \\
\hline GR-1 & $1.6 \pm 1.4$ & $1.1 \pm 1.1$ & 0.211 \\
\hline PI-0 & $1.3 \pm 0.3$ & $1.3 \pm 0.4$ & 0.966 \\
\hline PI-1 & $1.1 \pm 0.2$ & $1.1 \pm 0.2$ & 0.933 \\
\hline GI-0 & $1.3 \pm 0.3$ & $1.2 \pm 0.3$ & 0.607 \\
\hline GI-1 & $1.0 \pm 0.2$ & $0.9 \pm 0.2$ & 0.08 \\
\hline
\end{tabular}

Table 3: Mean difference in clinical parameters at baseline and six months between groups ( $\mathrm{n}=19$ for each group).

\begin{tabular}{|l|c|c|c|}
\hline \multicolumn{1}{|c|}{$\begin{array}{c}\text { Difference at } \\
\text { baseline and six months }\end{array}$} & Group & Mean value of difference \pm SD & P value \\
\hline \multirow{2}{*}{ PPD } & Control & $3.8 \pm 1.18$ & 0.005 \\
\hline \multirow{2}{*}{ CAL } & Test & $5.2 \pm 1.61$ & 0.001 \\
\hline GR & Control & $2.7 \pm 1.67$ & 0.39 \\
\hline PI & Test & $4.3 \pm 1.06$ & 0.91 \\
\hline GI & Control & $-0.42 \pm 0.51$ & $-0.58 \pm 0.61$ \\
\hline
\end{tabular}

\section{DISCUSSION}

The results of this study indicated that surgical therapy in open flap debridement alone and OFD with bovine-derived xenograft along with collagen membrane led to a clinically significant PPD reduction and CAL gain in patients with chronic periodontitis but statistically significant only in case of CAL at six months across both groups. Sites with initial PPD (5 to $10 \mathrm{~mm}$ ) showed a mean PPD reduction in Test Group from $7.9 \mathrm{~mm}$ to $2.7 \mathrm{~mm}$, difference was 5.2 $\mathrm{mm}$ from baseline to six months time, whereas in Control Group mean PPD reduction was from $7.0 \mathrm{~mm}$ to $3.2 \mathrm{~mm}$, difference was $3.8 \mathrm{~mm}$. Whereas mean gain in CAL in Test group was from $7.6 \mathrm{~mm}$ to $3.3 \mathrm{~mm}$ difference was $4.3 \mathrm{~mm}$ and in Control group it was from $7.3 \mathrm{~mm}$ to 4.6 mm difference was $2.7 \mathrm{~mm}$ which was almost twice more than the gain in Control group. So IBD treated with OFD along with $\mathrm{BDX}+\mathrm{CM}$ have clinically as well as statistically significant higher clinical attachment gain compared to open flap debridement alone.

Mean value of difference between the clinical parameters as PPD and CAL at baseline and six months showed statistically significant difference which helps in drawing the inference that IBD treated with OFD along with BDX+CM have improved healing compared to OFD alone.

Various investigations have been done for the evaluation of bone grafts and collagen membrane in adjunct to surgical therapy for regenerative procedure. Sculean et al. ${ }^{7}$ had a similar study where they compared the treatment of deep intrabony defects with bovine-derived xenograft and a bioresorbable collagen membrane to open flap debridement. Twenty-eight patients were studied for one year. At one year after therapy, the test group showed a reduction in mean PPD from 9.27 to $3.97 \mathrm{~mm}$, equivalent to $5.30 \mathrm{~mm}$ and a change in mean CAL from 10.27 to $6.27 \mathrm{~mm}$ equivalent to $4 \mathrm{~mm}$ and in the Control group, the mean PPD was reduced from 9.07 to $5.27 \mathrm{~mm}$ equivalent to $3.80 \mathrm{~mm}$ and the mean CAL changed from 10.57 to $8.47 \mathrm{~mm}$ equivalent to 2.10 $\mathrm{mm}$. Test group had statistically higher PPD reductions and CAL gains than the Control group. The results obtained in the current study was similar to this study as in current study also in test group there was PPD reduction of $5.2 \mathrm{~mm}$ whereas in Control group it was $3.8 \mathrm{~mm}$ and CAL gain was 
$4.3 \mathrm{~mm}$ in test group and $2.7 \mathrm{~mm}$ in Control group. Study by Ratka-Kruger 8 on guided tissue regeneration procedure with bioresorbable membranes versus conventional flap surgery, also resulted significant improvement of attachment levels, probing depths, as well as plaque index and bleeding on probing without any statistically significant differences which is similar to this present study. In the present study also, there was no statistically significant difference in Plaque Index, Gingival Index, PPD reduction, and CAL gain when compared between the two groups at baseline and six months except CAL at six months. Stavropoulos and Karring9 showed the similar result with the improvements in periodontal conditions after GTR treatment with or without the adjunct use of DBBM in six yrs follow ups.

As all the factors responsible for outcome of surgical treatment such as exclusion of smokers, regular monitoring of oral hygiene, infection Control. single operator were considered, the gain in CAL in test group most likely was due to the type of treatment and use of biomaterials. Postoperative radiograph in (Figure 10) shows bone fill with the use of BDX and CM which is suggestive of regeneration and this might be the reason of high CAL gain in test group and contributed by the use of bovine-derived xenografts and reabsorbable GTR membrane10 but this cannot be proved until histologic study is done. Histologic data from humans have shown that healing by regeneration are obtained with bone grafts..$^{5,7}$

In this study, bone grafts with GTR is used in the defect with the intention to support and stabilize the membrane which prevents the collapse of membrane and promotes bone healing and bone graft being osteoconductive forms a scaffold to support new bone formation from the osteoblastic cells. Human histologic case reports suggest that use of Bio-Oss grafts with resorbable membranes produces some amount of true periodontal regeneration with the formation of new cementum with functionally oriented inserting collagen fibers on a previously denuded root surface, and bone regeneration. . $^{5,11}$

The result in the current study showed more PPD reduction and CAL gain in test group which is suggestive of formation of connective tissue attachment and is consistent with the result of the study by Camelo et al. ${ }^{5}$ and Mellonig et al. ${ }^{11}$

Open flap debridement usually results in healing by the formation of long junctional epithelium which is comparatively weaker than the original epithelium and very limited regeneration. ${ }^{12,13}$ Stavropoulos and Karring ${ }^{9}$ have shown in their study that use of GTR membrane along with bone graft results in regeneration of the intrabony defects. The mean CAL gain of $4.3 \mathrm{~mm}$ in Test group and $2.7 \mathrm{~mm}$ in Control group is in consistent with other study results. ${ }^{14,7}$ Luepke et al. $^{15}$ have concluded that there was better result with the combination therapy of resorbable barrier membrane and DFDBA than with resorbable barrier membrane alone.

\section{CONCLUSION}

Treatment of intrabony defects with both the surgical therapies resulted in clinically significant PPD and CAL gain within six months period. There was clinical as well as statistical significance in CAL gain with the site treated with bovine-derived xenografts and bioresorbable collagen membrane which adds to the evidence that surgical therapy with combined bone graft and GTR membrane have improved healing.

Although results have shown improved clinical parameters in OFD with BDX+CM compared to OFD alone at six months, further longitudinal studies with larger sample size and histologic studies are recommended. Future similar studies should be extended to include the effect of other regenerative materials such as emdogain and platelet rich fibrin in the treatment of intrabony defects.

\section{ACKNOWLEDGEMENT}

National Academy of Medical Sciences provided the grant for this study. We would like to thank Dr. Amita Pradhan and Dr. Sujaya Gupta for their support.

Conflict of Interest: None. 


\section{REFERENCES}

1. Page RC, Kornman KS. The pathogenesis of human periodontitis: An introduction. Periodontol 2000. 1997;14:9-11.

2. Cortellini P, Prato PG, Tonetti, M. The simplified papilla preservation flap. A novel surgical approach for the management of soft tissues in regenerative procedures. Int J Periodont Rest. 1999;19(6):589-99.

3. Bouchard P, Giovannoli JL, Mattout C, Davarpanah M, Etienne D. Clinical evaluation of a bioabsorbable regenerative material in mandibular class II furcation therapy. J Clin Periodontol. 1997;24:511-8.

4. Yamada S, Shima N, Kitamura H, Sugito H. Effect of porous xenographic bone graft with collagen barrier membrane on periodontal regeneration. Int J Periodont Rest. 2002;22:389-97.

5. Camelo M, Nevins ML, Schenk RK, Simion M, Rasperini G, Lynch SE, et al. Clinical, radiographic, and histologic evaluation of human periodontal defects treated with Bio-Oss and Bio-Gide. Int J Periodont Rest. 1998;18:321-31.

6. Richardson CR, Mellonig JT, Brunsvold MA, McDonnell HT, Cochran DL. Clinical evaluation of Bio-Oss: a bovine-derived xenograft for the treatment of periodontal osseous defects in humans. J Clin Periodontol. 1999;26:421-8.

7. Sculean A, Berakdar M, Chiantella GC, Donos N, Arweiler NB, Brecx M. Healing of intrabony defects following treatment with a bovinederived xenograft and collagen membrane. J Clin Periodontol. 2003;30:73-80.

8. Ratka-Kruger P, Neukranz E, Raetzke P. Guided tissue regeneration procedure with bioresorbable membranes versus conventional flap surgery in the treatment of infrabony periodontal defects. J Clin Periodontol. 2000;27:120-7.

9. Starvopoulos A, Karring T. Guided tissue regeneration combined with a deproteinized bovine bone mineral (Bio-Osss) in the treatment of intrabony periodontal defects: 6-year results from a randomized-controlled clinical trial. J Clin Periodontol. 2010;37(2):200-10.

10. Cortellini P, Carnevale G, Sanz M, Tonetti MS. Treatment of deep and shallow intrabony defects. A multicenter randomized controlled clinical trial. J Clin Periodontol. 1998;25(12):981-7.

11. Mellonig JT. Human histologic evaluation of a bovine-derived bone xenograft in the treatment of periodontal osseous defects. Int J Periodontics Restorative Dent. 2000;20(1):19-29.

12. Caton J, Nyman S, Zander H. Histometric evaluation of periodontal surgery. II. Connective tissue attachment levels after four regenerative procedures. J Clin Periodontol. 1980;7(3):224-31.

13. Sculean A, Donos N, Brecx M, Reich E, Karring T. Treatment of intrabony defects with enamel matrix proteins and guided tissue regeneration. An experimental study in monkeys. J Clin Periodontol. 2000;27:466-72.

14. Sculean A, Windisch P, Chiantella GC, Donos N, Brecx M, Reich E. Treatment of intrabony defects with enamel matrix proteins and guided tissue regeneration. A prospective controlled clinical study. J Clin Periodontol. 2001;28:397-403.

15. Luepke PG, Mellonig JT, Brunsvold MA. A clinical evaluation of a bioresorbable barrier with and without decalcified freeze-dried bone allograft in the treatment of molar furcations. J Clin Periodontol. 1997;24(6):440-6. 